\title{
A notable bounded probability distribution for environmental and lifetime data
}

\author{
Hassan S. Bakouch ${ }^{1}$, Tassaddaq Hussain ${ }^{2}$, Christophe Chesneau ${ }^{3}$ and Tamás Jónás ${ }^{4}$ \\ ${ }^{1}$ Department of Mathematics, Faculty of Science, Tanta University, Tanta, Egypt, \\ Email: hassan.bakouch@science.tanta.edu.eg \\ ${ }^{2}$ Mirpur University of Science and Technology (MUST), 10250 Mirpur (AJK),Pakistan, \\ Email: tafkho2000@gmail.com \\ ${ }^{3}$ Université de Caen, LMNO, Campus II, Science 3, 14032, Caen, France, \\ Email: christophe.chesneau@unicaen.fr \\ ${ }^{4}$ Faculty of Economics, Eötvös Loránd University, Budapest, Hungary, \\ Email: jonas@gtk.elte.hu
}

\begin{abstract}
In this article, we introduce a notable bounded distribution based on a modification of the epsilon function which creates an upper bound on the domain of the distribution. Further, a key feature of the distribution links the readers with the asymptotic connections with the famous Lindley distribution, which is a weighted variant of the exponential distribution and also a mixture of exponential and gamma distributions. In some ways, the proposed distribution provides a flexible solution to the modeling of bounded characteristics that can be almost well-fitted by the Lindley distribution if the domain is restricted. Moreover, we have also explored its application, particularly with reference to lifetime and environmental points of view, and found that the proposed model exhibits a better fit among the competing models. Further, from the annual rainfall analysis, the proposed model exhibits a realistic return period of the rainfall.

Keywords: Epsilon distribution, Lindley distribution, Practical analysis, Applications, Hydrological measure.

AMS Subject Classification: 60E05, 62E15, 62F10.
\end{abstract}

\section{Introduction}

While observing real life phenomena, one usually comes across finite range of changes. Such finite changes generally give rise to bounded domain distributions. Among these bounded distributions, an upper bound is very helpful in analysing the annual stream flow and annual rainfall data (see Phien and Ajirajah (1984)). The most common bounded domain distributions are the uniform, power, Bates, arcsin, Kumaraswamy, Topp-Leone, beta, triangular, raised cosine, and Von Mises distributions. As an alternative to these distributions, Dombi et al. (2018) recently introduced the epsilon distribution (EpD). Mathematically, it is based on the epsilon function defined by

$$
\varepsilon_{\lambda, d}(x)= \begin{cases}\left(\frac{d+x}{d-x}\right)^{\lambda \frac{d}{2}}, & \text { if }-d<x<d, \\ 0, & \text { otherwise }\end{cases}
$$


where $\lambda \in \mathbb{R}, \lambda \neq 0, d>0$. This function is derived from the first-order epsilon differential equation and has the property of satisfying the following exponential limit property: For any $x \in(-d,+d)$, if $d \rightarrow \infty$, then $\varepsilon_{\lambda, d}(x) \rightarrow e^{\lambda x}$. Hence, a continuous random variable $X$ is said to have an epsilon distribution with the parameters $\lambda>0$ and $d>0$ if its cumulative distribution function (CDF) is given by

$$
F_{\lambda, d}^{*}(x)= \begin{cases}0, & \text { if } x \leq 0 \\ 1-\varepsilon_{-\lambda, d}(x), & \text { if } 0<x<d \\ 1, & \text { if } x \geq d\end{cases}
$$

As a result, the epsilon distribution is a bounded domain distribution with two parameters and satisfies the following limiting property: $\lim _{d \rightarrow+\infty} F_{\lambda, d}^{*}(x)=F_{\lambda}^{*}(x)$, where $F_{\lambda}^{*}(x)$ is the CDF of the exponential distribution with parameter $\lambda$. Among the applications, according to Dombi et al. (2018), the epsilon distribution can be used to describe the mortality and useful life cycle in the sense of reliability management under the assumption of a typical bathtub-shaped failure rate.

In this paper, we propose a notable two-parameter distribution also based on the epsilon function but connected to the famous Lindley distribution, instead of the exponential distribution. The Lindley distribution has a plural interest. First, it was created by Lindley $(1958,1965)$. It was first coined to express the distinction between fiducial and posterior distributions, and it has been widely used in mathematical theory and practice in recent years. Let us recall that the cumulative density function (CDF) of the Lindley distribution with parameter $\lambda>0$ is given by

$$
F_{\lambda}^{o}(x)= \begin{cases}0, & \text { if } x \leq 0 \\ 1-\left(1+\frac{\lambda x}{1+\lambda}\right) e^{-\lambda x}, & \text { if } x>0 .\end{cases}
$$

The Lindley distribution has been used to analyze large amounts of data, especially in the context of stress resistance and reliability modeling. There is a substantial literature on the Lindley distribution. Let us mention the Lindley distribution's dominance over the exponential distribution of banking customers' waiting times until service, as highlighted by Ghitany et al. (2008), the Lindley distribution's applications in lifetime data in the context of competing risks, as presented by Mazucheli and Achcar (2011), and a comparison study of the adequacy of exponential and Lindley distributions, as presented by Shanker et al. (2015) and Shanker and Mishra (2013), among others.

By capturing the idea of the epsilon distribution and adapting it to reach the Lindley distribution as a limit, we motivate the use of the following function:$$
F_{\lambda, d}(x)= \begin{cases}0, & \text { if } x \leq 0 \\ 1-\left(1+\frac{\lambda}{1+\lambda} \frac{d x}{d-x}\right) \varepsilon_{-\lambda, d}(x), & \text { if } 0<x<d \\ 1, & \text { if } x \geq d\end{cases}
$$

where $\lambda>0$ and $d>0$. The distribution defined by $F_{\lambda, d}(x)$ is called the epsilon-Lindley distribution (EpLD). Then, one can prove that it is a CDF, which satisfies $\lim _{d \rightarrow+\infty} F_{\lambda, d}(x)=F_{\lambda}^{o}(x)$; the Lindley distribution is a limit case of the EpLD, which is a rare property for a bounded support distribution. Furthermore, the related functions, such as the probability density function (PDF) and hazard rate function (HRF) are very flexible in their behaviour, as shown later. More precisely, by using a graphical analysis, the PDF adopt various shapes, like skewed to the right with J-shapes as well as an upside down U-shape. In all these cases, we observe a positive skewness and leptokurtic nature of the curve, which clearly indicates that it is designed to model the heavy-tailed phenomenon. Such 
phenomena are generally common in reliability applications, queuing theory and environmental aspects. In this regard, we focus on the environmental aspect and also lifetime direction. Our application section will help the reader to reach a decision to forecast the next generation's future in a better way. Environmental data analysis is based on the most efficient bounded models. One can mention the three parameter log normal distribution, generalized extreme value type II distribution, generalized extreme value type III distribution, three parameter gamma distribution, and three parameter log-Pearson distribution, among others. The non-closed form of the CDF in these popular hydrological models is a fundamental flaw, whereas the suggested model is based on only two parameters and has a closed form of its basic functions, including its CDF, which makes the determination of the return period considerably easier.

Further, the HRF adopts various shapes, from bathtub to increasing failure rate with a left skewed J-shape. This functional flexibility is a real plus for the EpLD from the modeling viewpoint. On the mathematical plan, the EpLD is a weighted version of the EpD. This weighted version not only models ascertainment biases but also a linear combination of probability distributions. We thus develop the statistical features offered by the EpLD through diverse aspects, including theoretical and practical facts. The practical lines interested in fitting, modeling and analysis of lifetime and environmental data are outlined by the proposed model. Further, from the annual rainfall analysis, we found a realistic return period of the rainfall by the proposed model.

The organization of the paper is as follows. Section 2 presents some other functions of interest in the EpLD like moments and parameter estimation. Section 3 covers the application area of the proposed model. Section 4 deals with conclusion and closing comments about the proposed distribution.

\section{Some Related Functions, Properties with Estimation}

\section{$2.1 \quad$ Related Functions}

We now illustrate the shape behavior of the main functions of the EpLD. First, let us focus on the CDF as defined in Equation (1). Figure 1 presents some graphs of this CDF for several values of the parameters.
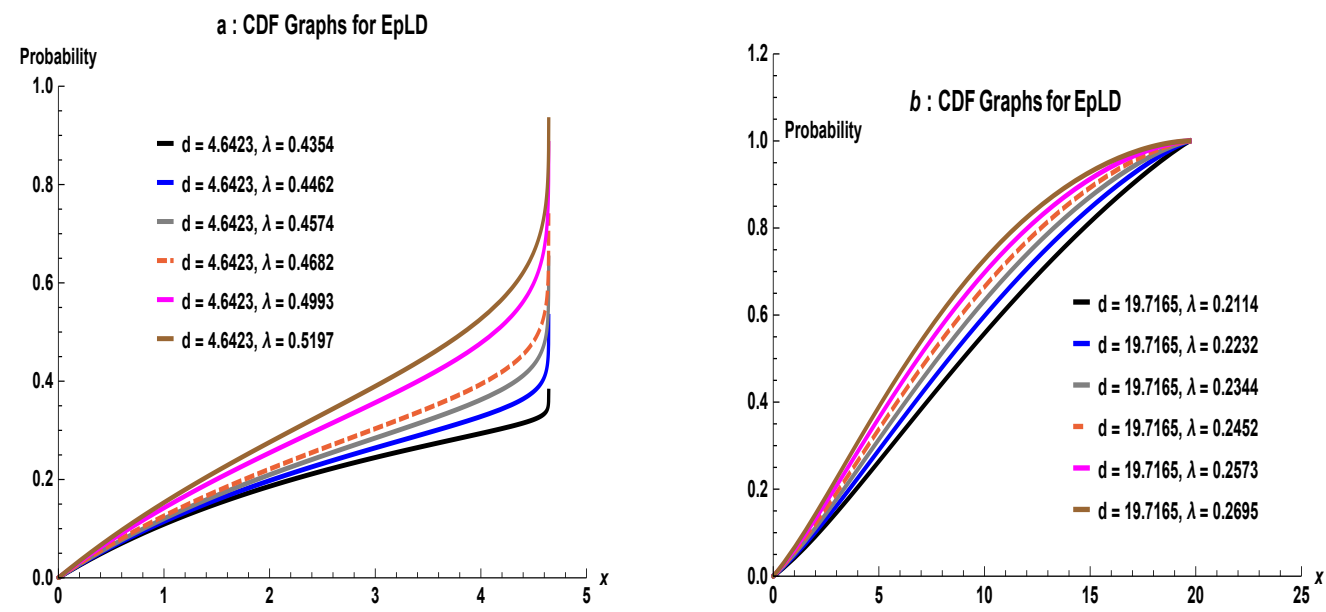

Figure 1: Graphs of CDF of the EpLD

Figure 1 depicts that for smaller values of both $d$ and $\lambda$, the convergence of CDF to 1 is very 
slow compared to that for larger $d$ and $\lambda$ values.

Let us now focus on the related PDF. The PDF of the EpLD is expressed as

$$
f_{\lambda, d}(x)= \begin{cases}\frac{\lambda d^{2}}{(1+\lambda)\left(d^{2}-x^{2}\right)}\left[1+\lambda-\frac{d+x-\lambda d x}{d-x}\right] \varepsilon_{-\lambda, d}(x), & \text { if } 0<x<d, \\ 0, & \text { otherwise. }\end{cases}
$$

Figure 2 presents some graphs of this PDF for several parameter values.
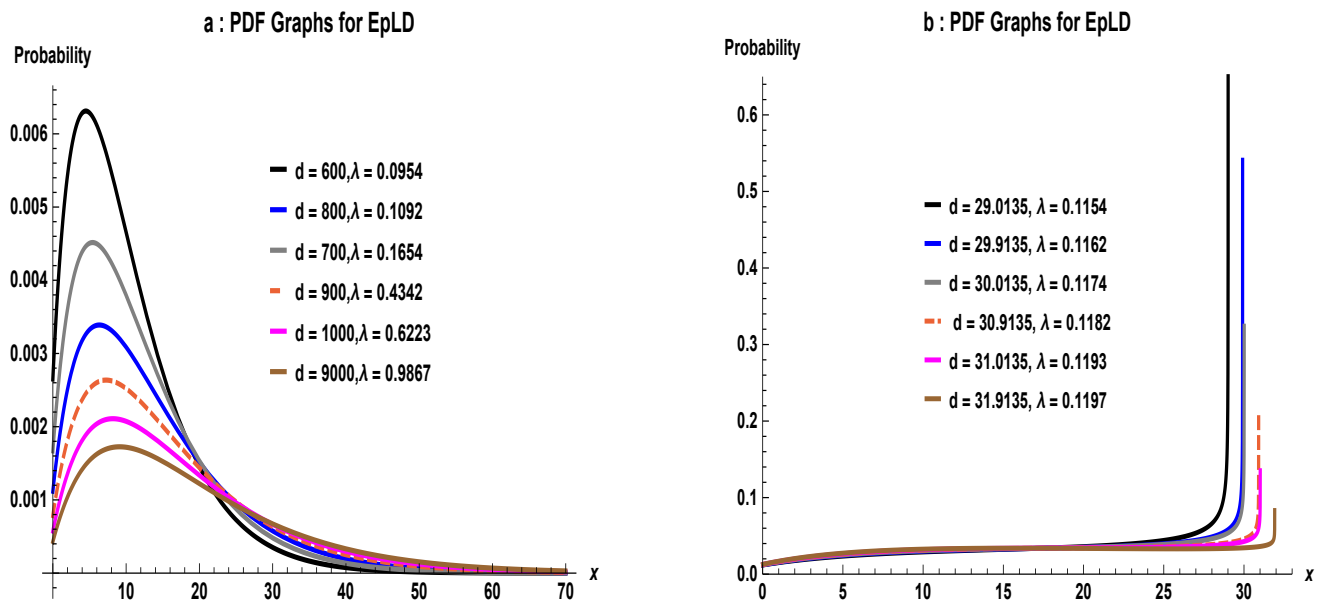

Figure 2: Graphs of PDF of the EpLD; unimodal shapes skewed to the right and J shapes
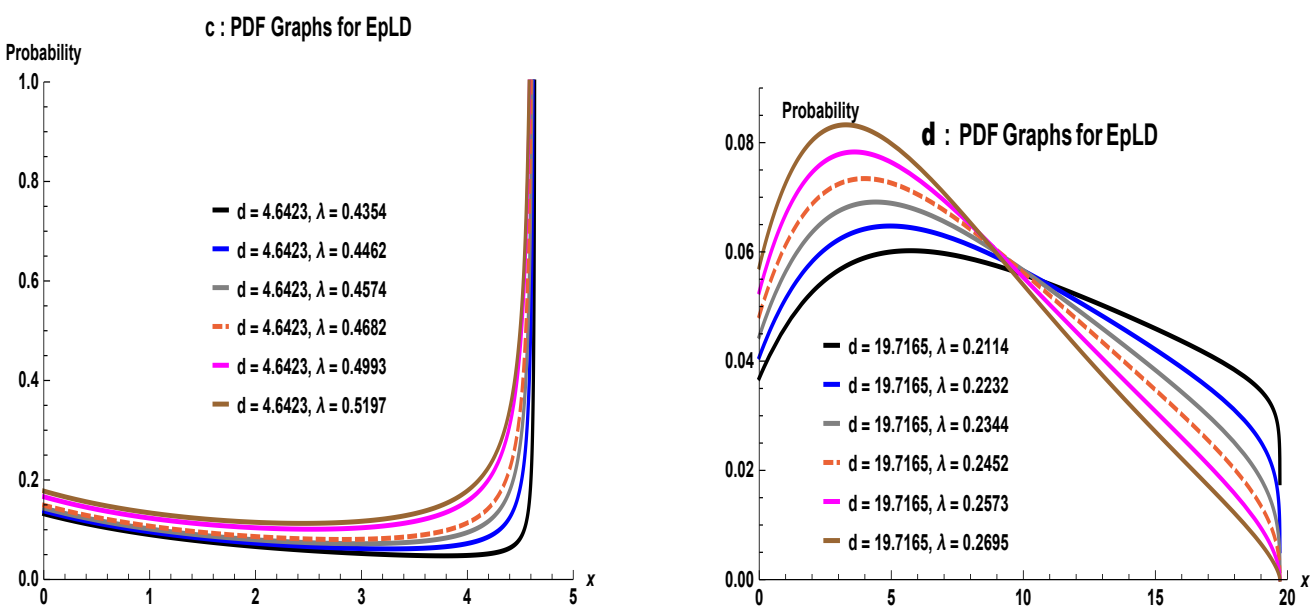

Figure 3: Graphs of PDF of the EpLD; U-J shapes and upside down U-shapes

From Figures 2 and 3, we see that the PDF of the EpLD also adopts various shapes, like unimodal shapes skewed to the right, various J-shapes, as well as an upside down U-shapes. In particular, Figure 2-b indicates that when the rate parameter $\lambda$ decreases, it increases the probability of events whatever the boundary value of $x$, i.e., $d$ is. Thus, the PDF of the EpLD distribution is extremely flexible, and, as developed in the introductive section, motivates the use of the EpLD for various modelling purposes, including the heavy-tailed phenomenon. 
In addition to its practical ability, the PDF of the EpLD has interesting mathematical decompositions. Indeed, one can view $f_{\lambda, d}(x)$ as follows:

- It is a weighted version of the PDF of the EpD, because it can be written as $f_{\lambda, d}(x)=$ $w_{\lambda, d}(x) f_{\lambda, d}^{*}(x)$, where

$$
w_{\lambda, d}(x)=1-\frac{d+x-\lambda d x}{(1+\lambda)(d-x)}
$$

and $f_{\lambda, d}^{*}(x)$ refers to the PDF of the epsilon distribution.

- Assuming that $\lambda>2 / d$, by noticing that

$$
\frac{d+x-\lambda d x}{(1+\lambda)(d-x)}=\frac{1}{1+\lambda}\left[\frac{\lambda}{4}(d-x)+\left(1-\frac{\lambda}{4}(d+x)\right) \frac{d+x}{d-x}\right]
$$

we can also write $f_{\lambda, d}(x)$ as a linear combination of PDFs and lenght biased PDFs of the EpD as

$$
\begin{aligned}
f_{\lambda, d}(x) & =\left(1-\frac{\lambda}{4(1+\lambda)}(d-x)\right) f_{\lambda, d}^{*}(x)-\frac{\lambda d}{(1+\lambda)(\lambda d-2)}\left(1-\frac{\lambda}{4}(d+x)\right) f_{\lambda-2 / d, d}^{*}(x) \\
& =\left(1-\frac{\lambda d}{4(1+\lambda)}\right) f_{\lambda, d}^{*}(x)-\frac{\lambda d}{(1+\lambda)(\lambda d-2)}\left(1-\frac{\lambda d}{4}\right) f_{\lambda-2 / d, d}^{*}(x) \\
& +\frac{\lambda}{4(1+\lambda)} x f_{\lambda, d}^{*}(x)+\frac{\lambda^{2} d}{4(1+\lambda)(\lambda d-2)} x f_{\lambda-2 / d, d}^{*}(x) .
\end{aligned}
$$

This expansion is useful to determine several probabilistic quantities related to the EpLD. As a major reliability function of the EpLD, the HRF is specified as

$$
h_{\lambda, d}(x)= \begin{cases}\frac{\lambda d^{2}}{(1+\lambda)\left(d^{2}-x^{2}\right)}\left[1+\lambda-\frac{d+x-\lambda d x}{d-x}\right]\left(1+\frac{\lambda}{1+\lambda} \frac{d x}{d-x}\right)^{-1}, & \text { if } 0<x<d, \\ 0, & \text { otherwise. }\end{cases}
$$

Figure 4 provides some graphs of this HRF for selected values of the parameters.
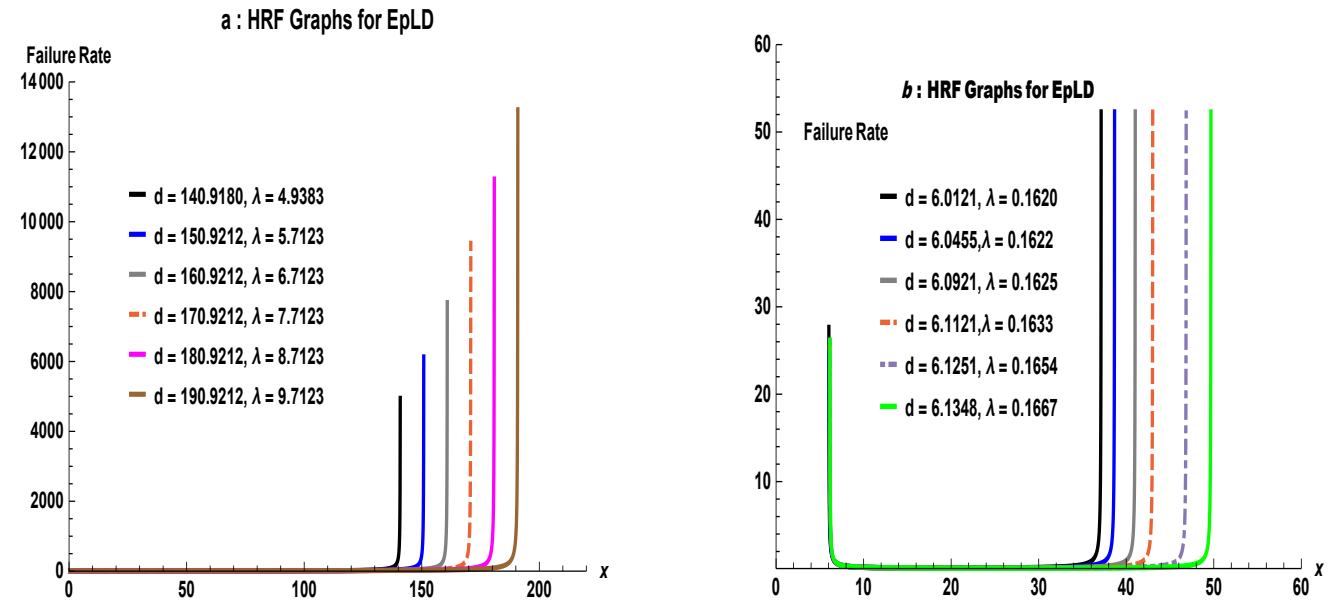

Figure 4: Graphs of HRF of the EpLD 
The graphs in Figure 4 clearly portray the HRF behaviour like increasing and bathtub shape in an impressive way.

We end this part by discussing the quantile analysis of the EpLD. As we know, in traditional probability and statistics as well as in stochastic analysis, the quantile function (QF) deals with a valuable way of describing a static or vigorous distribution, as a result, knowing how to use this function indicates certain advantages not available straight from the CDF or PDF. For example, the simplest way of simulating any non-uniform random variable is by applying its QF to uniform deviates. Similarly, from an environmental point of view, these functions usually help environmental scientists to calculate the return period and return level of any distribution.

In view of above, the $\mathrm{QF}$ of the EpLD, denoted by $Q_{\lambda, d}(u)$ with $u \in(0,1)$, is the solution of the following non-linear equation:

$$
\left(1+\frac{\lambda}{1+\lambda} \frac{d Q_{\lambda, d}(u)}{d-Q_{\lambda, d}(u)}\right) \varepsilon_{-\lambda, d}\left[Q_{\lambda, d}(u)\right]=1-u .
$$

To evaluate $Q_{\lambda, d}(u)$ at given $u$ and parameters, it is clear that mathematical software is required.

\subsection{Moments}

In mathematics and statistics, the word moments of a function are reckonable procedures associated to the shape of the function's graph. If the function represents density or mass function, then the first moment represent the center of the mass or expected value, and the second moment is the rotational inertia or the variance.So, the moments about the origin of the EpLD can be determined by using the expansion in Equation (3). For a random variable $Y_{\lambda, d}$ following the epsilon distribution with parameters $\lambda$ and $d$ and a random variable $X$ following the EpLD, the $r$-th moment of $X$ can be obtained as

$$
\begin{aligned}
\mu_{r}^{\prime}=E\left(X^{r}\right) & =\left(1-\frac{\lambda d}{4(1+\lambda)}\right) E\left(Y_{\lambda, d}^{r}\right)-\frac{\lambda d}{(1+\lambda)(\lambda d-2)}\left(1-\frac{\lambda d}{4}\right) E\left(Y_{\lambda-2 / d, d}^{r}\right) \\
& +\frac{\lambda}{4(1+\lambda)} E\left(Y_{\lambda, d}^{r+1}\right)+\frac{\lambda^{2} d}{4(1+\lambda)(\lambda d-2)} E\left(Y_{\lambda-2 / d, d}^{r+1}\right) .
\end{aligned}
$$

The the $r$-th and $(r+1)$-th moments of $Y_{\lambda, d}$ are well established, see Okorie and Nadarajah (2019). In a similar way, we can express the incomplete moments of $X$ in terms of incomplete moments of $Y_{\lambda, d}$. Thus, the mean and variance of $X$ can be obtained.

Similarly, the ratio of third mean moment to the square of second mean moment are the skewness and the ratio fourth moment about mean to second moment about mean is the kurtosis. Moreover, these moments are not only determine the shape of a function but also help to characterize the probability function. From these moments we are now able to interpret the shape and kurtosis behaviour of the EpLD. In this regard, Figure 5 portrays that the proposed model can exhibit versatile shapes ranging from negative to positive behaviour. In addition, the distribution can also exhibit the leptokurtic, mesokurtic and platykurtic behaviour which are evident in Figure 5 . 

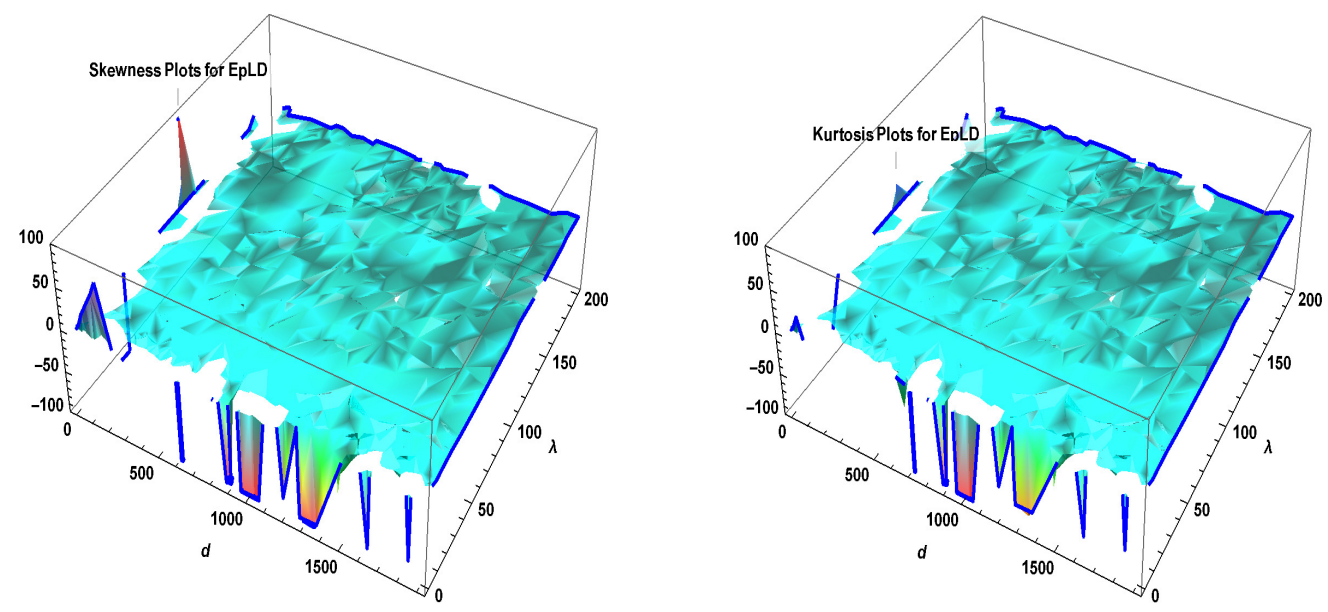

Figure 5: Graphs of skewness and kurtosis of the EpLD

\subsection{Parameters estimation}

Due to the importance of statistical inference, here we adopt the maximum likelihood method (MLEs) for estimation purpose which is the most commonly employed method. MLEs are designed to follow the regularity conditions which are usually helpful for constructing the confidence intervals and the test statistics. For these estimators, the large sample theory yields straightforward approximations that works well in finite samples. In order to achieve better approximation distributions, statisticians frequently strive to estimate quantities, such as the distribution of a test statistic that depends on the sample size. The resulting MLE approximation in distribution theory can be handled analytically or numerically with ease. Only complete samples are used to calculate the maximum likelihood estimates (MLEs) of the EpLD parameters. In this regard, let $x_{1}, \ldots, x_{n}$ be a realization of a random sample of size $n$ from the EpLD given by Equation (2). Then the log-likelihood function of the EpLD is given by

$$
\begin{aligned}
\ell(\lambda, d) & =2 n \log d+n \log \lambda-n \log (1+\lambda)-\left(1+\frac{d \lambda}{2}\right) \sum_{i=1}^{n} \log \left(\frac{d+x_{i}}{d-x_{i}}\right) \\
& +\sum_{i=1}^{n} \log \left[d \lambda+x_{i}((d-1) \lambda-2)\right]-3 \sum_{i=1}^{n} \log \left(d-x_{i}\right) .
\end{aligned}
$$

The log-likelihood can be maximized either directly by using the Mathematica [12.0] or by solving the nonlinear likelihood equations obtained by differentiating Equation (4).

$$
\frac{\partial \ell(\lambda, d)}{\partial \lambda}=\frac{n}{\lambda}-\frac{n}{1+\lambda}-\frac{d}{2} \sum_{i=1}^{n} \log \left(\frac{d+x_{i}}{d-x_{i}}\right)+\sum_{i=1}^{n} \frac{d+(d-1) x_{i}}{d \lambda+((d-1) \lambda-2) x_{i}} .
$$

The MLE of the parameter $\lambda$ is obtained by solving the nonlinear system $\partial \ell(\lambda, d) / \partial \lambda=0$. Since this equation cannot be solved analytically, so we prefer to use statistical packages like Mathematica [12.0]. For this purposes we used Global MLEs of the proposed model which take Lindley distribution MLEs as seed value. However, we have observed that we cannot obtain the estimate of $d$ from Equation (4). In order to obtain MLE of $d$ we adopted the methodology as, since $d$ is free from $x$ and it is the upper limit in the domain of $x$, so we suppose that $x_{(1)}, x_{(2)}, \ldots, x_{(n)}$, 
denotes the ordered sample corresponding to $x_{1}, x_{2}, \ldots, x_{n}$ this implies that the MLE for $d$ as $\hat{d}=\max \left(x_{1}, x_{2}, \ldots, x_{n}\right)+v$, where $v>0$ denotes an arbitrary constant. However, when we start to estimate a parameter, $d$ of a distribution using the sample, we undertake that all the elements in the sample are in the domain of the random variable. This is because the sample should be comprised of independent observations on the random variable in demand. Since the value of parameter $d$ determines the domain of attraction of the random variable that has a EpLD, in the estimation, it is a necessity that $d$ is greater than the maximal element in the sample. So we are looking for the robust value of $\hat{d}$ which will probably be established under the condition that $\hat{d}$ is greater than the largest element in the sample. Moreover, the convergence of proposed model's MLEs depends upon the huge value of $\hat{d}$, i.e., $\hat{d} \rightarrow \infty$ which follows the standard epsilon distribution theory, for more details, the reader is referred to Dombi et al. (2018), Dombi et al. (2019), Dombi and Jónás (2020) and Dombi and Jónás (2021).

\section{Model compatibility and its Application to Real-World Data}

In this section, we will concentrate on the modelling process's model selection and model validation. However, model selection is a challenging task and the prime of a suitable model and it is produced with the use of well-considered judgement based on whatever information is available. It is essential that the chosen model should be malleably sufficient to model the confronted data amply, while considering the settlement between simplicity of evaluation and the intricacy of the model.

Moreover, outstanding devotion must be waged to modeling behavior for large and small values of the variable of interest. In this regard, the modelling process includes validating the model, which includes various goodness-of-fit tests and graphical procedures. These statistical techniques for assessing hypothesised models are known as goodness-of-fit tests. An unsatisfactory fit, either analytical or graphical, may occur for the following reasons: i) The model is incorrectly specified. ii) The model specification is correct, but unfortunately carries a huge bias. In general, validation demands additional data, other information, and further testing as well as careful assessment of the fallouts.

\subsection{Goodness-of-Fit Tests}

As in such tests, researchers usually make a null hypothesis, $\mathrm{H}_{0}$ : The given data comes from a CDF with a specified form. For this purpose, we have considered four tests. The first test is the most famous $\chi^{2}$ test (Chi Square), due to Karl Pearson. It includes grouping observed data into intervals and may be used to assess the fit of data to any specified distribution (continuous or discrete). When using this test, a sample of size $n$ is assumed, with each observation falling into one of $k$ potential classifications. The observed and expected frequencies in the interval $i$ are denoted by $o_{i}$ and $e_{i}$, respectively. The test statistic is

$$
\chi^{2}=\sum_{i=1}^{k} \frac{\left(o_{i}-e_{i}\right)^{2}}{e_{i}} .
$$

However, this test has the advantages of being easy to apply and being applicable even when parameters are unknown (see Murthy, et al. (2004)). In addition, this test is considered a powerful test that is why it is not of much use in small or sometimes even modest size samples (see Murthy, et al. (2004)). The next three tests are based on the empirical cumulative distribution function (ECDF) and hence are often referred to as ECDF tests. 


\subsection{Kolmogorov-Smirnov (KS) Test}

The Kolmogorov-Smirnov (KS) test is grounded on the ECDF. Given $n$ ordered observations $Z_{1}, Z_{2}, \ldots, Z_{k}$, then the ECDF is defined as $\mathrm{E}_{k}=m_{i} / k$ where $m_{i}$ is the number of points less than $Z_{i}$ and the $Z_{i}$ are ordered from smallest to largest value. At the value of each ordered data point, this step function rises by $1 / k$. The greatest distance between the hypothesised CDF and $\mathrm{ECDF}$ is the test statistic KS. The mathematical expression of $\mathrm{KS}$ is given by

$$
\mathrm{KS}=\max _{1 \leq i \leq k}\left\{\frac{i}{k}-z_{i}, z_{i}-\frac{i-1}{k}\right\}
$$

where $z_{i}$ is the theoretical cumulative distribution of the distribution being tested. Other goodnessof-fit tests, like the Anderson-Darling test and the Cramér Von-Mises test, are alternatives of the KS test. As these modified tests are usually measured to be more powerful than the conventional KS test, many analysts prefer them.

\subsection{Anderson-Darling $\left(\mathrm{AD}_{0}^{*}\right)$ Test}

The Anderson-Darling $\left(\mathrm{AD}_{0}^{*}\right)$ test is an alternative of the $\mathrm{KS}$ test and usually attaches more weight to the tails than the KS test. Its test statistic is

$$
A_{0}^{*}=\left(\frac{2.25}{k^{2}}+\frac{0.75}{k}+1\right)\left\{-k-\frac{1}{k} \sum_{i=1}^{k}(2 i-1) \log \left(z_{i}\left(1-z_{k-i+1}\right)\right)\right\} .
$$

\subsection{Cramér-von Mises $\left(\mathrm{CVM}_{0}^{*}\right)$ Test}

$\mathrm{CVM}_{0}^{*}$ test is also a modification of the KS test. Which is usually considered to be more powerful than the original KS test. The $\mathrm{CVM}_{0}^{*}$ test is expressed as

$$
W_{0}^{*}=\sum_{i=1}^{k}\left(z_{i}-\frac{2 i-1}{2 k}\right)^{2}+\frac{1}{12 k} .
$$

A relative comparison of the selection of these tests indicates that: i) The ECDF tests are more powerful than the $\chi^{2}$ test. ii) The KS test is the most well-known ECDF test, but it is often much less powerful than the other ECDF tests $\left(\mathrm{AD}_{0}^{*}\right.$ and $\mathrm{CVM}_{0}^{*}$ tests) (see Murthy, et al. (2004)). Moreover, we have also applied information criteria for model selection purposes, such as Akaike information criterion (AIC), Bayesian information criterion (BIC), corrected Akaike information criterion (AICc), Hannan-Quinn information criterion (HQIC) and consistent Akaike information criterion (CAIC). The following are the definitions of AIC, AICc, HQIC, and CAIC:

$$
\begin{gathered}
\mathrm{AIC}=2 \hbar-2 l, \quad \mathrm{AICc}=\mathrm{AIC}+\frac{2 \hbar(\hbar+1)}{n-\hbar-1}, \quad \mathrm{BIC}=\hbar \log (n)-2 l, \\
\mathrm{HQIC}=-2 l+\hbar \log (\log (n)), \quad \mathrm{CAIC}=-2 l+\frac{2 \hbar n}{n-\hbar-1},
\end{gathered}
$$

where $l$ denotes the estimate of the maximm log-likehood function, $\hbar$ is the number of parameters to be estimated and $n$ is the number of data.

Along with these model selection procedures, we have also used the Kullback-Leibler information criterion philosophy and applied the Vuong test proposed by Vuong (1989). 


\subsection{Vuong Test}

It is a closeness test based on the likelihood-ratio-based test for model selection using the KullbackLeibler information criterion philosophy. This test may be used for non-nested models, and it generally compares the null hypothesis that the two competing models are equally near to the actual data against the alternative that one model performs better. Further discussion about Vuong statistics can be found in Hussain et al. (2019).

\subsection{Competing Models}

We compare the proposed model with the following well known models: epsilon probability distribution (EpD) (see Dombi et al. (2018)), two parameter Lindley (TPLD) distribution (see Shanker et al. (2015)), A quasi Lindley distribution (QLD) (see Shanker and Mishra (2013)), Lindley distribution (LD) (see Lindley (1958)) and exponential distribution (ED). For the sake of transparency, these competitors are defined by the following PDFs:

- for the EpD:

$$
f_{\lambda, d}^{E p D}(x)=\frac{\lambda d^{2}}{d^{2}-x^{2}} \varepsilon_{-\lambda, d}(x), \quad 0<x<d,
$$

and $f_{\lambda, d}^{E p D}(x)=0$ for $x \notin[0, d]$, with $d>0$,

- for the TPLD:

$$
f_{\lambda, d}^{T P L D}(x)=\frac{\lambda^{2}(1+d x)}{d+\lambda} e^{-\lambda x}, \quad x>0,
$$

and $f_{\lambda, d}^{T P L D}(x)=0$ for $x \leq 0$, with $\lambda>0$ and $d>-1$,

- for the QLD:

$$
f_{\lambda, d}^{Q L D}(x)=\frac{\lambda(d+\lambda x)}{d+1} e^{-\lambda x}, \quad x>0,
$$

and $f_{\lambda, d}^{Q L D}(x)=0$ with $\lambda>0$ and $d>-1$,

- for the LD:

$$
f_{\lambda}^{L D}(x)=\frac{\lambda^{2}(1+x)}{1+\lambda} e^{-\lambda x}, \quad x>0,
$$

and $f_{\lambda}^{L D}(x)=0$ for $x \leq 0$, with $\lambda>0$,

- for the ED:

$$
f_{\lambda}^{E D}(x)=\lambda e^{-\lambda x}, \quad x>0,
$$

and $f_{\lambda}^{E D}(x)=0$, with $\lambda>0$.

We consider four different real-world data sets. 


\subsection{Lifetime Data Sets}

Data sets I and II. The first and second data sets are taken from Walpole et al. (2012) and Andrews and Herzberg (1985), respectively. The first data represent the length of life in years, measured to the nearest tenth of 30 similar fuel pumps, while the second data represent the life of fatigue fracture of Kevlar 373 epoxy that is subjected to constant pressure at the 90 stress level until all have failed. The measurements of the first data set are 2.0, 3.0, 0.3, 3.3, 1.3, 0.4, 0.2, 6.0, $5.5,6.5,0.2,2.3,1.5,4.0,5.9,1.8,4.7,0.7,4.5,0.3,1.5,0.5,2.5,5.0,1.0,6.0,5.6,6.0,1.2,0.2$. The second data set measurements are given as: $0.0251,0.0886,0.0891,0.2501,0.3113,0.3451,0.4763$, 0.5650, 0.5671, 0.6566, 0.6748, 0.6751, 0.6753, 0.7696, 0.8375, 0.8391, 0.8425, 0.8645, 0.8851, 0.9113, 0.9120, 0.9836, 1.0483, 1.0596, 1.0773, 1.1733, 1.2570, 1.2766, 1.2985, 1.3211, 1.3503, 1.3551, 1.4595, $1.4880,1.5728,1.5733,1.7083,1.7263,1.7460,1.7630,1.7746,1.8275,1.8375,1.8503,1.8808,1.8878$, $1.8881,1.9316,1.9558,2.0048,2.0408,2.0903,2.1093,2.1330,2.2100,2.2460,2.2878,2.3203,2.3470$, $2.3513,2.4951,2.5260,2.9911,3.0256,3.2678,3.4045,3.4846,3.7433,3.7455,3.9143,4.8073,5.4005$, $5.4435,5.5295,6.5541,9.0960$. In this regard, we have compiled the descriptive statistics which are listed in Table 1 and the total time on test (TTT) plot introduced by Aarset (1987), which is portrayed in Figure 6 for Data sets I and II. In particular, the TTT plot indicates the empirical $\mathrm{HRF}$ and estimated behaviour of the HRF of the EpLD are very close to each other as compared to the competing models.

\begin{tabular}{cccccccc}
\hline Data set & Sample size & Mean & Median & Standard deviation & Skewness & Kurtosis & $\frac{\text { Skewness }}{\text { Kurtosis }}$ \\
\hline I & 30 & 2.7967 & 2.1500 & 2.2273 & 0.3412 & 1.5689 & 0.2175 \\
\hline II & 76 & 1.9592 & 1.5335 & 1.6753 & 1.9796 & 8.1608 & 0.2426 \\
\hline
\end{tabular}

Table 1: Descriptive statistics for Data sets I and II.
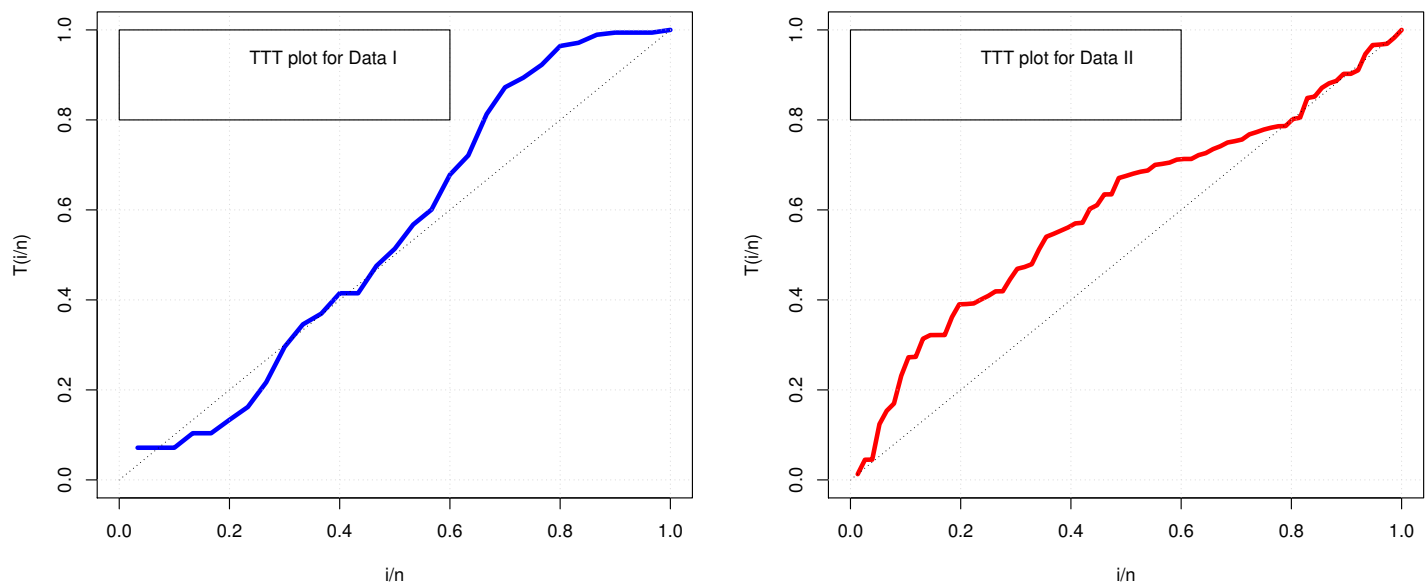

Figure 6: Estimated TTT plots of Data sets I and II 


\begin{tabular}{cccccccc}
\hline Data set & Sample size & Mean & Median & Standard deviation & Skewness & Kurtosis & $\frac{\text { Skewness }}{\text { Kurtosis }}$ \\
\hline I & 30 & 2.7802 & 2.2285 & 2.2505 & 1.1766 & 4.2477 & 0.2769 \\
\hline II & 76 & 1.9589 & 1.5739 & 1.7362 & 1.4309 & 5.4976 & 0.2602 \\
\hline
\end{tabular}

Table 2: Theoretical statistics from the EpLD

\subsubsection{Discussion and Analysis of Lifetime Data Sets}

Tables 1 and 2 reveal that theoretical and observed descriptive statistics show a remarkable closeness to each other and it seems that both data sets are being simulated by the proposed model.

\begin{tabular}{cccccccc}
\hline Distribution & $\hat{\lambda}$ & $\hat{d}$ & $\mathrm{CVM}_{0}^{*}$ & $\mathrm{AD}_{0}^{*}$ & $\mathrm{KS}$ & $\chi^{2}(d f)$ & $\mathrm{p}$-value \\
\hline EpLD & 0.5839 & 1219.9011 & 0.5059 & 0.0728 & 0.1408 & $1.0760(2)$ & 0.5839 \\
\hline EpD & 0.3575 & $6.31 \times 10^{6}$ & 0.7569 & 0.1189 & 0.1358 & $1.6246(2)$ & 0.4438 \\
\hline TPLD & 0.3575 & $-1.92 \times 10^{-26}$ & 0.7569 & 0.1189 & 0.1358 & $1.6246(2)$ & 0.4438 \\
\hline QLD & 0.5123 & 1.3097 & 0.5512 & 0.0809 & 0.1341 & $1.0990(2)$ & 0.5772 \\
\hline LD & 0.5834 & $\ldots$ & 0.5061 & 0.0729 & 0.1409 & $1.0767(2)$ & 0.5831 \\
\hline ED & 0.3576 & $\ldots$ & 0.7569 & 0.1189 & 0.1358 & $1.6246(2)$ & 0.4438 \\
\hline
\end{tabular}

Table 3: MLEs and goodness-of-fit statistics for Data set I

Note that as the parameter $d$ specifies the support of of the PDF of the EpLD in Equation (2), i.e., it is positive only if $x \in(0, d)$. This means that the value of parameter $d$ must meet the requirement $d>\max _{i=1,2, \ldots, n}\left(x_{i}\right)$, see Dombi et al. (2019). That is why we observed that $\hat{d}$ is large for any data set, as shown in the related tables. From the TTT-plot for Data set I, we can see that the curve has three characteristic phases: (1) a first convex phase, where the failure rate is decreasing; (2) a second quasi linear phase with a constant failure rate; (3) and a third concave phase, where the failure rate is increasing. That is, the TTT-plot for Data set I portrays a bathtub-shaped like failure rate curve. Noting the TTT-plot for Data set II, we can conclude that it exhibits an increasing failure rate phenomenon of the empirical failure rate function. Hence, both of the above data sets are efficiently modelled by the proposed model. Since $d$ is large, EpLD is almost identical to LD, and so they have almost the same goodness values. Such a suitability of the proposed model is reflected in Tables 3 and 4, where the EpLD yields a smallest value of the goodness of fit statistics along with highest p-vlaue for $\chi^{2}$ statistics. In addition, we have also accessed the performance of the model with respect to LD via the log-likelihood ratio test, which is usually applicable for nested models, which clearly indicates that. 


\begin{tabular}{cccccccc}
\hline Distribution & $\hat{\lambda}$ & $\hat{d}$ & $\mathrm{CVM}_{0}^{*}$ & $\mathrm{AD}_{0}^{*}$ & $\mathrm{KS}$ & $\chi^{2}(d f)$ & $\mathrm{p}$-value \\
\hline EpLD & 0.7948 & 99910.0267 & 1.4902 & 0.2667 & 0.1156 & $6.8497(6)$ & 0.3349 \\
\hline EpD & 0.5104 & 40763.1154 & 3.2134 & 0.5746 & 0.1663 & $13.4562(6)$ & 0.0363 \\
\hline TPLD & 0.5104 & $8.29 \times 10^{-10}$ & 3.0187 & 0.5746 & 0.1663 & $13.4562(6)$ & 0.0363 \\
\hline QLD & 0.9542 & 0.1499 & 0.6003 & 0.1016 & 0.1025 & $9.3621(8)$ & 0.3126 \\
\hline LD & 0.7947 & $\ldots$ & 1.4902 & 0.2668 & 0.1156 & $6.8496(6)$ & 0.3348 \\
\hline ED & 0.5104 & $\ldots$ & 3.0188 & 0.5746 & 0.1663 & $13.4562(6)$ & 0.0363 \\
\hline
\end{tabular}

Table 4: MLEs and goodness-of-fit statistics for Data set II

However, Tables 5 and 6 portray that QLD and LD yield minimum values of information criterion, which some seem to be a penalty of over parametrization, particularly with reference to the LD model. Previously we pointed out that the LD distribution may be viewed as an asymptotic EpLD distribution, i.e., if $d \rightarrow \infty$, then the EpLD distribution is identical to the LD distribution. We can observe a practical implication of this finding in the tables from Table 3-6. Namely, when a data set can be modelled well by the LD distribution, then it can also be modelled well by an EpLD distribution with a sufficiently large value of parameter $d$, and vice versa. Certainly, in such a case, the estimates of the $\lambda$ parameters and the corresponding goodness-of-fit statistics are very close.

\begin{tabular}{ccccccc}
\hline Distribution & $-l$ & AIC & AICC & BIC & HQIC & CAIC \\
\hline EpLD & 60.7645 & 125.529 & 125.973 & 128.331 & 123.977 & 125.973 \\
\hline EpD & 60.8525 & 125.705 & 126.149 & 128.507 & 124.153 & 126.149 \\
\hline TPLD & 60.8529 & 125.706 & 126.15 & 128.508 & 124.154 & 126.15 \\
\hline QLD & 60.4864 & 124.973 & 125.417 & 127.775 & 123.421 & 125.417 \\
\hline LD & 60.7668 & 123.534 & 123.676 & 124.935 & 123.982 & 123.676 \\
\hline ED & 60.8528 & 123.706 & 123.848 & 125.107 & 124.154 & 123.848 \\
\hline
\end{tabular}

Table 5: Estimate of the maximum log-likelihood and information criteria for Data set I

\begin{tabular}{ccccccc}
\hline Distribution & $-l$ & AIC & AICC & BIC & HQIC & CAIC \\
\hline EpLD & 123.674 & 251.348 & 251.512 & 256.009 & 250.279 & 251.512 \\
\hline EpD & 127.114 & 258.228 & 258.392 & 262.889 & 257.159 & 258.392 \\
\hline TPLD & 127.114 & 258.228 & 258.392 & 262.889 & 257.159 & 258.392 \\
\hline QLD & 121.65 & 247.3 & 247.464 & 251.961 & 246.231 & 247.464 \\
\hline LD & 123.675 & 249.35 & 249.404 & 251.681 & 250.281 & 249.404 \\
\hline ED & 127.114 & 258.228 & 258.392 & 262.889 & 257.159 & 258.392
\end{tabular}

Table 6: Estimate of the maximum log-likelihood and information criteria for Data set II

Furthermore, Table 7 also pleads for the suitability of the proposed model. But Vuong statistics show that QLD and LD are strong competitors for the proposed model. 


\begin{tabular}{ccccc}
\hline Models & Data set I & Suitability & Data set II & Suitability \\
\hline EpLD- EpD & 2.7872 & EpLD & 32.5790 & EpLD \\
\hline EpLD-TPD & 2.7872 & EpLD & 32.5804 & EpLD \\
\hline EpLD-QLD & -0.9287 & Indecisive & -15.3623 & QLD \\
\hline EpLD-LD & 1513.008 & EpLD & -1321291 & LD \\
\hline EpLD-ED & 2.7872 & EpLD & 32.5790 & EpLD \\
\hline
\end{tabular}

Table 7: Vuong test statistics for Data sets I and II

Furthermore, an analysis of the histogram's form revealed that we can observe from Figure 7 that the proposed model matches the data in a better way than competing models.
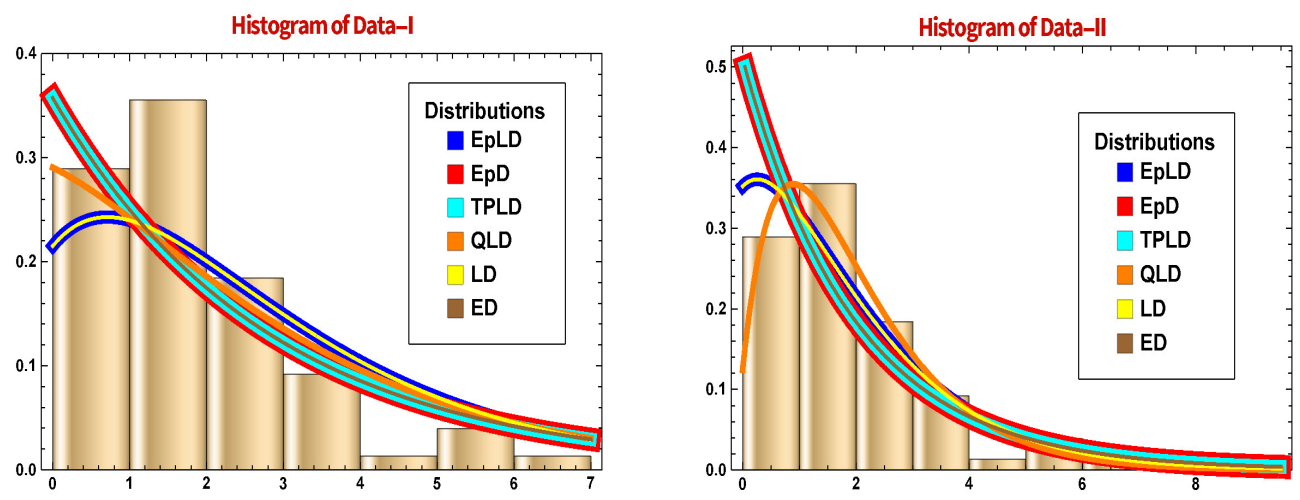

Figure 7: Data sets I and II fit via histograms

\subsection{Environmental Data Sets}

Data sets III and IV. The third and fourth data sets are the total amount of rain fall in mm of Pakistani cities Lasbella and Bunji which covers a period of 30 years (1981 to 2010) with 30 values of annual rain fall in each set. They were reported by Hussain et al. (2019). The third data set measurements are as follows: 138.11818, 89.5, 246.5, 142.6, 143.5, 47.4, 105.7, 182.6, 153.7, 119.9, $56.5,272.8,99.9,426.1,205.6,169.8,308.3,80.5,104.0,37.7,223.0,9.2$, 474.6, 25.3, 209.6, 182.5, 196.2, 254.9, 103.6, 117.6. The fourth data set contains the following rainfall measurements: 248.8 , $82.2,102.2,217.9,113.2,248.2,244.1,122.2,144.9,63.2,62.8,139,228.7,216.4,144.8,252.6,144.8$, $157.2,168.5,139.1,74.3,154.6,339.4,154.1,156.3,200.7,97.5,96.3,155.2,298.8$, respectively. The descriptive statistics of these data sets and corresponding theoretical statistics from the EpLD are presented in the Tables 8 and 9, respectively. Box-plots of the data are given in Figure 8.

\begin{tabular}{cccccccc}
\hline Data set & Sample size & Mean & Median & Standard deviation & Skewness & Kurtosis & $\frac{\text { Skewness }}{\text { Kurtosis }}$ \\
\hline III & 30 & 164.241 & 143.05 & 108.163 & 1.1201 & 4.2629 & 0.2627 \\
\hline IV & 30 & 165.6 & 154.35 & 70.3731 & 0.5800 & 2.7073 & 0.2142 \\
\hline
\end{tabular}

Table 8: Descriptive statistics for Data sets III and IV 


\begin{tabular}{cccccccc}
\hline Data set & Sample size & Mean & Median & Standard deviation & Skewness & Kurtosis & $\frac{\text { Skewness }}{\text { Kurtosis }}$ \\
\hline III & 30 & 163.952 & 138.302 & 114.483 & 1.0747 & 4.0723 & 0.2639 \\
\hline IV & 30 & 176.0789 & 160.9816 & 102.6899 & 0.5248 & 2.5549 & 0.2054 \\
\hline
\end{tabular}

Table 9: Theoretical statistics from the EpLD

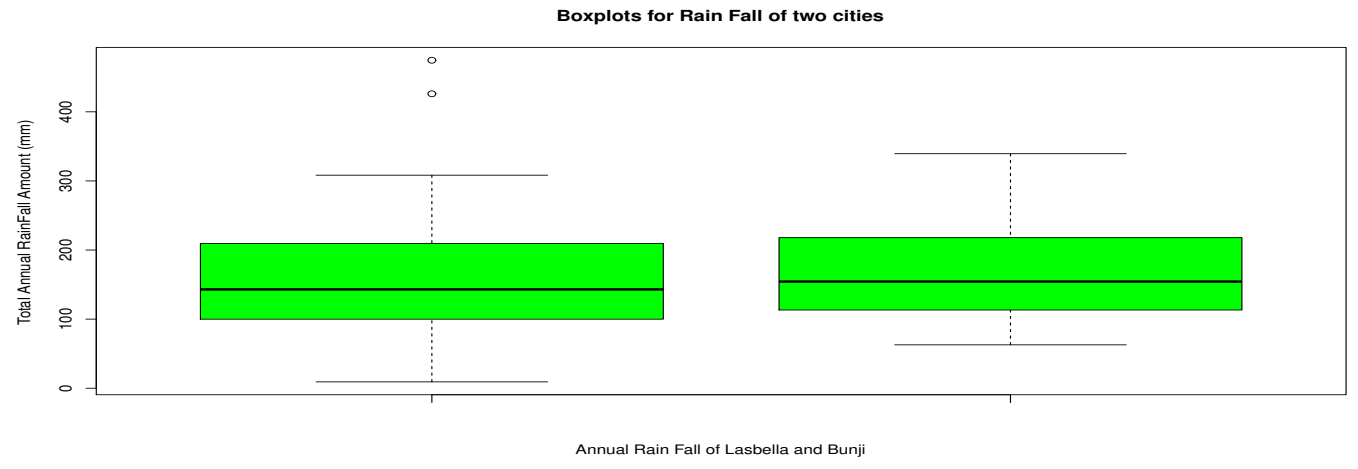

Figure 8: Box-plots for Data sets III and IV

In order to analyse the environmental data, we have also checked some features of the environmental data, namely homogeneity, independence and stationarity. For this purpose, we applied the Mann-Whitney (M-W) test for testing homogeneity and stationarity, and the Mann-Kendall (M-K) test for trend detection. In this regard, we have observed that both data sets accept the hypotheses of homogeneity and stationarity at a 5 percent level of significance with Z-scores of 0.3568 and -0.2777 , respectively. Similarly, the hypothesis of independence and identically distributed distribution is accepted at a 5 percent level of significance with Z-scores of 0.4817 and -0.6943 , respectively. For details of these tests, readers are referred to Haktanir et al. (2013).

\subsubsection{Analysis and Discussion of Environmental Data}

From Table 8 and Figure 8 as well as Table 9, it is obvious that the empirical and theoretical aspects of the data sets in the presence of outliers in Data set III are in close agreement and indicate that the model can effectively be used if the data are positively skewed and leptokurtic in nature, which are the obvious characteristics of environmental data. Such findings are further consolidated by viewing Tables 10 and 11 which portray that the EpLD exhibits minimum values of goodness of fit statistics. 


\begin{tabular}{cccccccc}
\hline Distribution & $\hat{\lambda}$ & $\hat{d}$ & $\mathrm{CVM}_{0}^{*}$ & $\mathrm{AD}_{0}^{*}$ & $\mathrm{KS}$ & $\chi^{2}(d f)$ & $\mathrm{p}$-value \\
\hline EpLD & 0.0122 & 8329.81 & 0.0472 & 0.2778 & 0.1117 & $0.2294(2)$ & 0.8916 \\
\hline EpD & 0.0041 & $9.29 \times 10^{6}$ & 1.8655 & 0.3667 & 0.2417 & $3.6401(2)$ & 0.1620 \\
\hline TPLD & 0.00411 & 0.0000 & 0.3667 & 1.8655 & 0.2417 & $3.6401(2)$ & 0.1620 \\
\hline QLD & 0.0121 & 0.0159 & 0.0479 & 0.2796 & 0.1122 & $0.2350(2)$ & 0.8891 \\
\hline LD & 0.0121 & $\ldots$ & 0.0473 & 0.2779 & 0.1121 & $0.2296(2)$ & 0.8915 \\
\hline ED & 0.0061 & $\ldots$ & 0.3549 & 1.8494 & 0.2224 & $3.3397(2)$ & 0.1883 \\
\hline
\end{tabular}

Table 10: MLEs and goodness-of-fit statistics for Data set III

\begin{tabular}{cccccccc}
\hline Distribution & $\hat{\lambda}$ & $\hat{d}$ & $\mathrm{CVM}_{0}^{*}$ & $\mathrm{AD}_{0}^{*}$ & $\mathrm{KS}$ & $\chi^{2}(d f)$ & $\mathrm{p}$-value \\
\hline EpLD & 0.0123 & 498.906 & 0.2002 & 1.3785 & 0.1650 & $2.5612(1)$ & 0.1096 \\
\hline EpD & 0.0060 & $9.25 \times 10^{7}$ & 0.8954 & 4.6593 & 0.3156 & $10.7892(1)$ & 0.0010 \\
\hline TPLD & 0.0060 & $6.56 \times 10^{-13}$ & 0.8954 & 4.6594 & 0.3156 & $10.3156(1)$ & 0.0013 \\
\hline QLD & 0.0121 & $-4.12 \times 10^{-27}$ & 0.3099 & 1.8943 & 0.1898 & $2.7965(1)$ & 0.0945 \\
\hline LD & 0.0120 & $\ldots$ & 0.3164 & 1.9301 & 0.1904 & $2.8808(1)$ & 0.0896 \\
\hline ED & 0.0060 & $\ldots$ & 0.8954 & 4.6593 & 0.3156 & $10.7892(1)$ & 0.0010 \\
\hline
\end{tabular}

Table 11: MLEs and goodness-of-fit statistics for data set IV

Tables 10 and 11 indicate that ECDF test statistics for goodness-of-fit tests are low, which ensures that the EpLD is a good competitor to QLD and LD.

However, likelihood aspects and information criterion values also favour the proposed model, which can be visualized in Tables 12 and 13, respectively.

\begin{tabular}{ccccccc}
\hline Distribution & $-l$ & AIC & AICC & BIC & HQIC & CAIC \\
\hline EpLD & 179.123 & 362.247 & 362.691 & 365.049 & 360.695 & 362.691 \\
\hline EpD & 183.04 & 370.08 & 370.524 & 372.882 & 368.528 & 370.524 \\
\hline TPLD & 183.04 & 370.08 & 370.524 & 372.882 & 368.528 & 370.524 \\
\hline QLD & 179.125 & 362.251 & 362.695 & 365.053 & 360.699 & 362.695 \\
\hline LD & 179.13 & 360.26 & 360.403 & 361.661 & 360.708 & 360.403 \\
\hline ED & 183.04 & 368.08 & 368.223 & 369.481 & 368.528 & 368.223 \\
\hline
\end{tabular}

Table 12: Estimate of the maximum log-likelihood and information criteria for Data set III 


\begin{tabular}{ccccccc}
\hline Distribution & $-l$ & AIC & AICC & BIC & HQIC & CAIC \\
\hline EpLD & 173.351 & 350.703 & 351.147 & 353.505 & 349.151 & 351.147 \\
\hline EpD & 183.287 & 370.575 & 371.019 & 373.377 & 369.023 & 371.019 \\
\hline TPLD & 183.287 & 370.575 & 371.019 & 373.377 & 369.023 & 371.019 \\
\hline QLD & 174.435 & 352.869 & 353.314 & 355.672 & 351.318 & 353.314 \\
\hline LD & 174.576 & 351.152 & 351.294 & 352.553 & 351.6 & 351.294 \\
\hline ED & 183.287 & 368.575 & 368.717 & 369.976 & 369.023 & 368.717
\end{tabular}

Table 13: Estimate of the maximum log-likelihood and information criteria for Data set IV

Furthermore, the shape of our proposed model, as shown in Figure 9, matches the data in a better way compared to competing models.
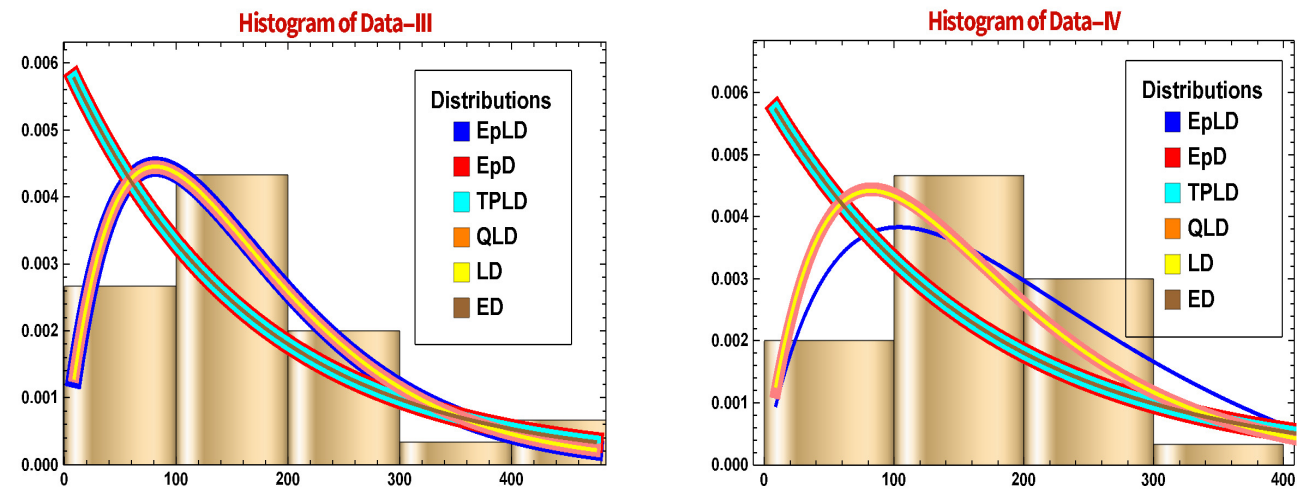

Figure 9: Data sets III and IV fit via histograms

Furthermore, Vuong statistics as depicted in Table 14 also show the capability of the proposed model.

\begin{tabular}{ccccc}
\hline Models & Data set III & Suitability & Data set IV & Suitability \\
\hline EpLD- EpD & 19.4212 & EpLD & 56.0414 & EpLD \\
\hline EpLD-TPD & 19.4213 & EpLD & 56.0412 & EpLD \\
\hline EpLD-QLD & 1954.888 & EpLD & 8.5923 & EpLD \\
\hline EpLD-LD & -39411 & LD & 9.8278 & EpLD \\
\hline EpLD-ED & 16.7418 & EpLD & 56.0414 & EpLD \\
\hline
\end{tabular}

Table 14: Vuong test statistics for Data sets III and IV

\subsubsection{Hydrological Parameters}

The annual series is very common in frequency analysis for two reasons. The earliest is its accessibility, as most data are managed in such a way that the annual series is commonly available. The other one is that there is a simple hypothetical basis for deducing the frequency of annual series data beyond the range of observation (see World Meteorological Organization (2009)). Moreover, we observed that both series are valid and approved by the M-W and M-K tests, as shown in the earlier section. 
Therefore, it can be settled that, according to the consequences of relevant tests, both the annual rainfall series documented at Lasbella (Pakistan) and Bounji (Pakistan) are homogeneous, independent, non-periodic and trend-free. Hence, classical frequency analyses are applied to all of the annual rainfall series. From the above mentioned analysis, we can conjecture that the EpLD is a suitable model for the above mentioned data sets, so we have decided to portray its return period for those interested in environmental data, which is being studied in the coming subsection.

\subsubsection{Return Period}

The average number of years in which an event is predicted to be equalled or exceeded only once is the return period $\mathfrak{T}$ of a particular level. The return period is the reciprocal of the probability of exceeding the threshold in a particular year (see World Meteorological Organization (2009)). The link between the annual return time and the exceedance probability may be stated as follows if the yearly exceedance probability is designated $1 / \mathfrak{T}$. Since the probability of exceedance is $P\left(X>x_{\mathfrak{T}}\right)=1 / \mathfrak{T}$, this implies a return level with a return period of $\mathfrak{T}=1 / \mathfrak{p}$ is a high threshold $x_{\mathfrak{T}}$ whose probability of exceedance is $\mathfrak{p}$.
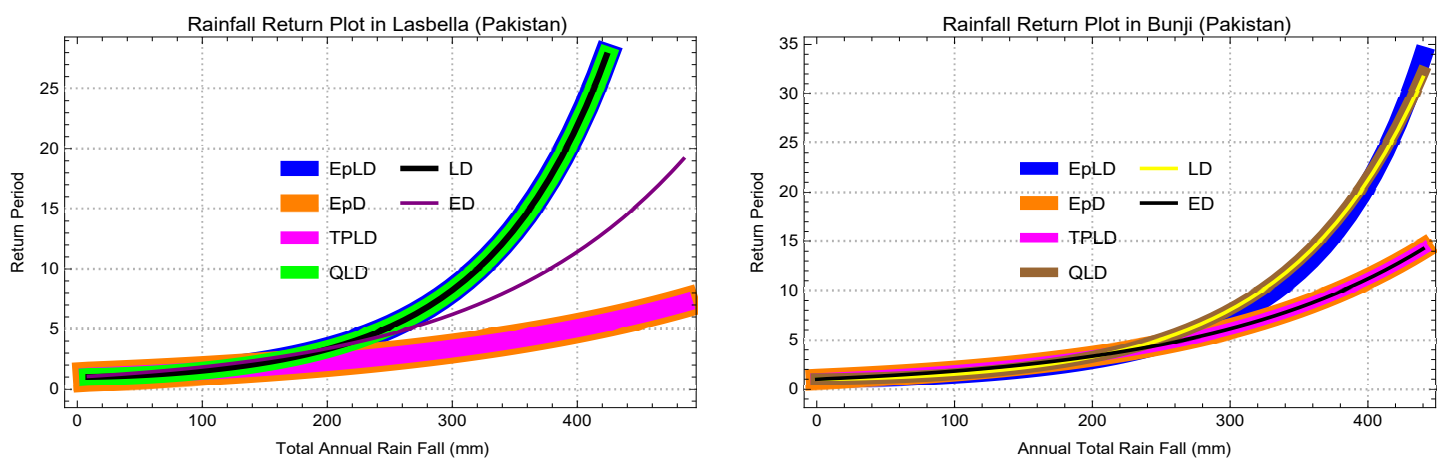

Figure 10: Return periods of the competing models for Data sets III and IV

In this regard, we have studied that the EpLD yields a realistic return period which can be visualized from Figure 10.

\section{Conclusion}

In this article, we proposed a notable bounded distribution under the name epsilon Lindley distribution (EpLD). This new distribution provides a flexible solution to the problem of modeling bounded characteristics. This feature of EpLD is based on the finding that this distribution can be treated as a bounded alternative of the well-known Lindley distribution. Here, we estimated the parameters of EpLD using the maximum likelihood method. Next, we studied the applications of the proposed distribution both in lifetime and environmental data modeling. Based on the empirical results, we could conclude that the proposed methodology works quite well. In particular, for the annual rainfall data set, the EpLD yields a realistic return period when compared with other competing models.

\section{Author contribution statement}

All authors contributed equally to the paper. 


\section{Conflicts of interest}

The authors declare no conflicts of interest.

\section{References}

Andrews, D.F. and Herzberg, A.M. (1985). Data: a collection of problems from many fields for the student and research worker.Springer, New York.

Aarset M. V. (1987). How to identify a bathtub hazard rate. IEEE Transactions on Reliability, 36(1), 106-108.

Dombi, J., Jónás, T. and Tóth, Z.E. (2018). The Epsilon Probability Distribution and its Application in Reliability Theory, Acta Polytechnica Hungarica, Vol. 15, No.1, pp 197 - 216.

Dombi, J., Jónás, T., Tóth, Z.E. and Árva, G. (2019). The omega probability distribution and its applications in reliability theory. Qual Reliab Eng Int., 35, 2, 600-626.

Dombi, J. and Jónás, T. (2020) On an alternative to four notable distribution functions with applications in engineering and the business sciences. Acta Polytech. Hung, 17, 231-252.

Dombi, J. and Jónás, T. (2021). Advances in the theory of probabilistic and fuzzy data scientific methods with applications. In Studies in Computational Intelligence; Springer: Berlin/Heidelberg, Germany, 814, 1-186.

Ghitany, M.E., Atieh, B. and Nadarajah, S. (2008). Lindley distribution and its Applications, Mathematical Computation and Simulation, 78, 4, 493-506.

Haktanir, T., Bajabaa, S. and Masoud, M. (2013). Stochastic analyses of maximum daily rainfall series recorded at two stations across the Mediterranean Sea. Arabian Journal of Geosciences, 6: 3943-3958. DOI: https : //doi.org/10.1007/s12517 - $012-0652-0$

Hussain, T., Bakouch, H.S. and Chesneau, C. (2019). A new probability model with application to heavy-tailed hydrological data. Environ Ecol. Stat 26, 127-151. Available at https : //doi.org/10.1007/s10651 - $019-00422-7$

Lindley, D.V. (1958). Fiducial distributions and Bayes theorem, Journal of the Royal Statistical Society, A, 20, 102-107.

Lindley, D.V. (1965). Introduction to Probability and Statistics from a Bayesian Viewpoint, Part II: inference, Cambridge University Press, New York.

Mazucheli, J. and Achcar, J.A. (2011). The Lindley distribution applied to competing risks lifetime data, Computer Methods Programs in Biomedicine, 104, 2, 188-192.

Murthy,D.N.P., Xie M., and Jiang, R. (2004). Weibull Models, y John Wiley and Sons, Inc., Hoboken, New Jersey.

Okorie, I. E. and Nadarajah, S. (2019). On the Omega Probability Distribution. Quality and Reliability Engineering International, 35, 6, 2045-2050.

Phien, H.N. and Ajirajah, T. J. (1984). Applications of the log Pearson type-3 distribution in hydrology. Journal of Hydrology, 73(3-4),359-372. DOI:https : //doi.org/10.1016/0022 1694(84)90008- 8 . 
Shanker, R. and Mishra, A. (2013). A quasi Lindley distribution, Biometrics \& African Journal of Mathematics and Computer Science Research, 6, 4, 64-71.

Shanker, R., Hagos, F. and Sujatha, S. (2015). On modeling of Lifetimes data using exponential and Lindley distributions, Biometrics \& Biostatistics International Journal, 2, 5, 1-9.

Vuong, Q. H. (1989). Likelihood ratio tests for model selection and non-nested hypotheses. Econometrica, 57(2), 307-333.

Walpole, R.E., Myers, R.H. and Myers, S.L. (2012). Probability and Statistics for Engineers and Scientists.Pearson Education, Inc, 501 Boylston Street, Suite 900, Boston.

World Meteorological Organization. (2009). Guide to Hydrological Practices Volume II Management of Water Resources and Application of Hydrological Practices WMO-No. $1686^{\text {th }}$, Chairperson, Publications Board World Meteorological Organization (WMO) 7 bis, avenue de la Paix P.O. Box 2300 CH-1211 Geneva 2, Switzerland. 